\title{
Philosophiques
}

\section{Le principe de Pascal-Hume et la métaphysique}

\section{Jean-René Vernes}

Volume 22, numéro 2, automne 1995

URI : https://id.erudit.org/iderudit/027330ar

DOI : https://doi.org/10.7202/027330ar

Aller au sommaire du numéro

Éditeur(s)

Société de philosophie du Québec

ISSN

0316-2923 (imprimé)

1492-1391 (numérique)

Découvrir la revue

Citer cet article

Vernes, J.-R. (1995). Le principe de Pascal-Hume et la métaphysique. Philosophiques, 22(2), 237-246. https://doi.org/10.7202/027330ar

\section{Résumé de l'article}

Le raisonnement par lequel Hume établit l'ori- gine empirique de Vidée de cause repose implicitement sur un principe de possibilité a priori et même de probabilité a priori, dont Hume n'a pas remarqué le caractère rationnel, tant il paraissait naturel. Ce principe est identique à celui sur lequel Pascal fonde le calcul des probabilités. Si l'on admet sa légitimité, il en résulte deux conséquences capita- les pour la théorie de la connaissance : 1 - La raison ne se limite pas aux seuls principes logiques en oeuvre dans la déduction mais doit reconnaître que ce qui est également pensable est également probable a priori. 2 - Ce principe de probabilité fournit une preuve de l'existence d'une réalité extérieure à la conscience, que l'on cherchait en vain dans le principe de causalité. 


\title{
LE PRINCIPE DE PASCAL-HUME ET LA MÉTAPHYSIQULE
}

\author{
par \\ Jean-René Vernes
}

RÉSUMÉ : Le raisonnement par lequel Hume établit l'origine empirique de l'idée de cause repose implicitement sur un principe de possibilité a priori et même de probabilité a priori, dont Hume n'a pas remarqué le caractère rationnel, tant il paraissait naturel. Ce principe est identique à celui sur lequel Pascal fonde le calcul des probabilités. Si l'on admet sa légitimité, il en résulte deux conséquences capitales pour la théorie de la connaissance:

1 - La raison ne se limite pas aux seuls principes logiques en ouvre dans la déduction mais doit reconnaître que ce qui est également pensable est également probable a priori.

2 - Ce principe de probabilité fournit une preuve de l'existence d'une réalité extérieure à la conscience, que l'on cherchait en vain dans le principe de causalité.

ABSTRACT: The reasoning by which Hume established the empirical origin of the idea of cause rests on a principle of a priori possibility and even a priori probability of which Hume did not notice the rational character, so natural did it seem. This principle is identical to the one on which Pascal founded the calculation of probabilities. If its legitimy is admitted, two important consequences follow for the theory of knowledge :

1 - Reason is not limited only to the logical principles at word in deduction but must recognize that, a priori, what is equally thinkable it equally probable.

2 - This principle of probability provides a proof of the existence of a reality outside the consciousness that one looked for in vain in the principle of causality. 
On comprend aisément l'admiration que les penseurs grecs, tel Platon, ont éprouvée pour la démonstration géométrique. Celle-ci possède une double qualité : elle permet à la fois d'expliquer et de prévoir.

Il semble que les Babyloniens aient connu mille ans avant Pythagore la propriété des triangles rectangles : $\mathrm{a}^{2}=\mathrm{b}^{2}+\mathrm{c}^{2}$.

Sans doute l'avaient-ils mesurée sur de nombreux triangles. Dès lors on peut imaginer un géomètre babylonien s'écriant : “c'est extraordinaire. J'ai dessiné ce matin vingt triangles rectangles, tous différents les uns des autres, des grands, des petits, des triangles dont les angles à la base étaient presque égaux ou, au contraire, très dissemblables et tous possédaient cette même propriété. N'est-ce pas là une coïncidence surprenante ?"

La démonstration de Pythagore écarte l'idée de coïncidence pour y substituer la nécessité. L'explication réside dans cette substitution. La question "pourquoi » suppose la diversité des possibles. Là où n'existe qu'un seul possible le besoin d'expliquer disparaît.

Mais la nécessité n'éclaire pas seulement l'expérience passée, elle garantit l'expérience future. La démonstration de Pythagore nous rassure. Tous les triangles qui se présenteront dans notre expérience - tous les triangles rectangles euclidiens, préciserions-nous aujourd'hui - posséderont la propriété de Pythagore. L'avenir ne saurait se soustraire à la nécessité qui a régi le passé.

\section{L'absence de nécessité}

Malheureusement notre expérience ne se soumet que pour une part infime à la nécessité géométrique. Non seulement bien des données de la conscience sont étrangères à la spatialité, les sons, les odeurs, les émotions par exemple. Mais les images visuelles elles-mêmes, qui constituent le lieu par excellence de la géométrie, ne sont géométrisables que pour un seul de leurs aspects, infiniment pauvre au regard de leur richesse perceptive. La qualité se refuse à la quantification des géomètres, la qualité, c'est-à-dire le bleu, le jaune, le rouge et l'innombrable diversité des apparences sensibles marquée par toutes les nuances de la couleur et de la luminosité.

Or nulle nécessité ne lie entre elles les apparences sensibles. Elles s'étendent selon des plages plus ou moins larges qui durent à travers le temps. Nous y reconnaissons des objets doués d'étendue et de permanence, mais ce n'est là rien de plus qu'un fait, nous n'y voyons pas de nécessité. Rien ne nous interdit de les imaginer sous mille formes différentes, s'associant et se succédant sans ordre et sans loi. La liberté de l'imagination s'oppose à la nécessité géométrique. Hume fondera sur cette constatation sa critique de la causalité.

\section{La critique de l'idée de cause}

Les objets, suggère Descartes, ne nous sont connus que comme des collections d'apparences sensibles. Si révolutionnaire qu'elle soit, cette cons- 
tatation va s'imposer à la pensée moderne. Mais dans un premier temps la conception du monde ne s'en trouvera pas bouleversée. Ces apparences sensibles ne doivent-elles pas avoir une cause? Quelle cause plus naturelle leur supposer que des objets matériels, doués d'une existence extérieure à la perception que nous en avons? La dualité fondamentale de la matière et de la pensée se trouve confirmée.

C'est apparemment la critique humienne de l'idée de cause qui va faire prendre à la philosophie moderne un tournant capital. En montrant que nous n'avons pas de connaissance a priori de la causalité, Hume ruine l'argument qui nous permettait de passer de la conscience vécue à l'affirmation d'objets matériels doués d'une existence autonome. Et il semble que depuis cette date aient échoué toutes les tentatives qui prétendaient donner la preuve d'une réalité ontologique. S'il en est bien ainsi, la pensée n'est-elle pas la seule réalité ? C'est dans la pensée qu'il faudrait trouver l'explication du monde.

\section{Le principe implicite de la philosophie moderne}

Cette conception moderne de la philosophie est toutefois intimement liee à une conviction implicite. C'est que le seul lien rationnel que nous puissions découvrir entre les objets de la pensée est de nature déductive. Tel est le principe de la démonstration géométrique. Mais que pouvons-nous déduire d'une perception qui est de nature qualitative ? Les données sensibles échappent à la déduction. Elles ne nous permettent pas de conclure à l'existence de quoi que soit qui leur serait extérieur.

La critique de la philosophie moderne est subordonnée à une remise en question du privilège d'exclusivité accordé à la rationalité déductive. La raison se réduit-elle à lier entre elles des propositions conformément aux principes de la déduction ? Selon la réponse que l'on donne à cette question, la conception du monde à laquelle on aboutit sera fondamentalement différente.

En 1654 Pascal énonce les premiers développements rigoureux du calcul des probabilités, esquissé seulement un siècle plus tôt par Cardan. Son principe essentiel peut se formuler à partir d'un exemple concret : si l'on considère un dé bien fait, c'est-à-dire parfaitement cubique et formé d'une matière homogène, la probabilité d'apparition de chacune des six faces est la même, lorsqu'on jette le dé dans des conditions convenables.

En s'inspirant de ce principe une science nouvelle va se constituer qui aboutit un siècle et demi plus tard au célèbre Traité des probabilités de Laplace. Celui-ci y définit la probabilité comme le rapport entre le nombre des cas favorables et le nombre total des cas possibles, supposés équiprobables. Plus exactement Laplace écrit dans son « premier principe» : «la probabilité [...] est le rapport du nombre des cas favorables à celui de tous les cas 
possibles » puis dans son « second principe » : « cela suppose les cas également possibles" ${ }^{1}$ ".

C'est seulement à partir de ce moment, au cours du XIX ${ }^{e}$ siècle qu'on soulève un problème nouveau : comment peut-on justifier le calcul des probabilités ? On reproche à Laplace sa définition de la probabilité. N'est-ce pas commettre un cercle que de définir celle-ci à partir de cas également possibles? La probabilité n'est jamais donnée directement dans l'expérience. La seule chose que celle-ci nous révèle, ce sont des fréquences. On constate seulement que quand un événement aléatoire se répète indéfiniment, par exemple le jet d'un dé, sa fréquence tend vers une limite de plus en plus précise. C'est celle-ci qui constituerait la probabilité de l'événement.

À travers cette controverse apparaît une opposition fondamentale entre deux conceptions des rapports de la pensée et du réel. Si Laplace définit la probabilité à partir de cas équiprobables, c'est que cette dernière notion lui apparaît douée d'une évidence immédiate. Les six faces d'un dé bien fait sont équivalentes au regard de la pensée, elles sont interchangeables entre elles. L'équiprobabilité n'est que la conséquence expérimentale de cette équivalence intellectuelle.

Dans la conception de Laplace, l'équiprobabilité est perçue comme une propriété de la pensée, le pouvoir que nous avons de nous représenter indifféremment les six faces d'un dé bien fait. Le problème fondamental est de savoir si nous pouvons conclure de cette équivalence intellectuelle aux résultats effectifs de l'expérience sensible.

Un problème analogue pourrait être posé quant à la notion de nécessité. Le théorème de Pythagore ne démontre pas directement que tout triangle rectangle possède nécessairement la propriété de Pythagore. Il démontre seulement qu'il en est ainsi pour tout triangle pensé. Un triangle qui ne satisferait pas à cette propriété est impensable. En conclure qu'il est impossible relève d'une proposition distincte, ce qui est impensable est impossible ou ce qui est nécessaire au regard de la pensée est nécessaire pour l'être.

Lorsque Laplace pose en principe fondateur du calcul des probabilités l'équiprobabilité des six faces d'un dé bien fait, il suppose implicitement un principe analogue mais différent : ce qui est également pensable est également possible. Le caractère commun aux deux principes réside dans l'assimilation affirmée entre l'être et la pensée. Leur différence consiste en ce que dans un cas l'assimilation porte sur la nécessité et dans l'autre sur la possibilité.

Or justifier le passage de la nécessité pensée à la nécessité réelle est relativement aisé. Il en est autrement de celui qui relie le pensable au possible. La conception empiriste de la probabilité est issue de cette difficulté. Il en résulte que l'on refuse au calcul des probabilités un statut identique à celui de la démonstration géométrique. Il convient d'analyser les conséquences de ce refus.

1. Pierre-Simon Laplace, Essai philosophique sur les probabilités, Paris, Gauthier-Villars, 1921, p. 9. 


\section{L'explication des événements aléatoires}

Admettons à titre provisoire la conception empiriste des événements aléatoires. Comment alors les expliquer?

Les fréquences tendent vers une limite, la fréquence d'apparition du 6 avec un dé bien fait tend vers 1 sur 6 . Voilà un fait qui est non seulement reconnu par la théorie empiriste, mais qui lui sert de point de départ. L'existence même de ce fait conduit à poser deux problèmes :

1) Pourquoi la fréquence d'apparition du 6 tend-elle vers une limite qui ne varie pas avec le temps ? On pourrait parfaitement imaginer que pendant une période déterminée, le mois de février d'une certaine année par exemple, cette fréquence soit très faible, tandis qu'elle augmenterait considérablement pendant le mois suivant. Or cela ne se produit pas. Pourquoi ?

2) Une fois admise l'existence d'une limite des fréquences, on peut se demander pourquoi cette limite est précisément de 1 sur 6 et non par exemple de 1 sur 4 pour le chiffre 6 et de 1 sur 18 pour le chiffre 4 . Il est difficile de ne pas voir dans le fait que les fréquences limites des six faces sont égales à une forme de rationalité.

Mais les difficultés de l'empirisme ne s'arrêtent pas là. En fait Pascal a élaboré ses calculs de probabilité sans recourir à l'expérience. Il se fonde sur des principes a priori. Or ses calculs ont été confirmés par les expériences ultérieures, dans la mesure où des prévisions de probabilité peuvent être confirmées tout au moins de façon approximative. Nous nous retrouvons dans la situation imaginaire de notre géomètre babylonien. Peut-on expliquer la concordance des prévisions et des expériences par une simple coïncidence particulièrement heureuse?

Force est de constater que le calcul des probabilités est avec la géométrie le seul domaine où la pure réflexion ait permis de prévoir l'expérience. Dénier au principe d'équiprobabilité un caractère a priori, c'est renoncer simultanément à trouver une explication à l'existence de fréquences limites et à la concordance entre l'expérience et la prévision. Si le calcul des probabilités avait été inventé avant la géométrie, il est vraisemblable qu'il aurait suscité le même enthousiasme et qu'il aurait engendré une conception de la raison très différente de celle qui est aujourd' hui généralement admise.

\section{Le principe sous-jacent de la philosophie humienne}

Plus intéressante cependant encore, parce qu'elle semble ne jamais avoir été faite, est la remarque suivante : l'un des plus grands philosophes empiristes des temps modernes, David Hume, a fondé sa pensée sur un principe a priori identique à celui de Pascal.

Rappelons, en effet, le passage sans doute le plus caractéristique des textes dans lesquels Hume montre l'origine empirique de la notion de cause : "quand je vois, par exemple, une bille de billard qui se meut en ligne droite 
vers une autre $[\ldots]$ ne puis-je pas concevoir que cent événements différents pourraient aussi bien suivre de cette cause ? Les billes ne peuvent-elles toutes deux rester en repos absolu ? La première bille ne peut-elle retourner en ligne droite ou rebondir de la seconde dans une ligne ou une direction quelconque ? Toutes ces conceptions sont cohérentes et concevables. Alors, pourquoi donner la préférence à l'une d'elles, qui n'est ni plus cohérente ni plus concevable que les autres ${ }^{2}$ ?

Ce texte appelle les remarques suivantes :

1) Hume constate que nous pouvons imaginer les deux boules de billard décrivant après le choc une multitude de trajets différents.

2) Avant que nous ayons fait l'expérience, nous ignorons lequel de ces trajets sera suivi par les deux boules.

3) Nous devons donc avant l'expérience considérer ces différents trajets comme possibles et même comme également possibles, car nous n'avons aucune raison de privilégier l'un ou l'autre.

4) Hume admet donc qu'il existe une notion a priori du possible qui s'identifie au pensable : tout ce qui est pensable est possible a priori. Bien plus, ce qui est également pensable est a priori également possible.

Nous voici très exactement devant le principe de Pascal. Pour ce dernier les six faces d'un dé bien fait sont équivalentes au regard de la pensée, elles peuvent également être représentées. Elles doivent donc être considérées comme également possibles a priori. Hume applique le même raisonnement aux différents trajets que peuvent suivre les boules de billard. Ils peuvent être également représentés. Ils doivent donc être considérés comme également possibles.

Cette similitude entre les conceptions de Pascal et de Hume est capitale et mérite d'être soulignée. C'est pourquoi je propose d'appeler principe de Pascal-Hume le principe dans lequel elle s'exprime : ce qui est également pensable est également possible a priori.

La différence entre Pascal et Hume est cependant, elle aussi, capitale. Elle tient au fait que le possible a priori de Pascal se réalise dans l'expérience, tandis que celui de Hume s'y trouve constamment démenti. C'est évidemment cette différence qui a masqué la similitude de leur principe. C'est également elle qui a empêché Hume de prendre conscience que sa démonstration reposait en dernière analyse sur un principe a priori. Hume a suivi une démarche progressive, il a cherché les conséquences, qu'entrainait son analyse de la notion de cause. Il a négligé la démarche régressive, qui aurait consisté à chercher ses présupposées implicites. La démarche de Pascal était, au contraire, strictement progressive, puisque son raisonnement partait explicitement du principe implicite de la pensée de Hume.

2. David Hume, Enquête sur l'entendement humain, Traduction André Leroy, Paris, Aubier-Montaigne, 1947, p. 75 . On trouve un texte très voisin dans le Traité de la nature humaine, idem, 1946, p. 189. 


\section{La synthèse de Pascal et de Hume}

Si l'on rapproche maintenant l'Univers expérimental de Pascal, à savoir une succession d'événements aléatoires qui se succèdent sans ordre apparent (les résultats d'un jet de dé) et de l'Univers de Hume, où les chocs de deux boules de billard sont suivis d'effets constants, il convient d'en faire la synthèse.

Cette synthèse s'est opérée spontanément dans la pensée collective autour de la notion de loi, car les lois physiques avaient dans l'expérience quotidienne une importance sans commune mesure avec les événements aléatoires de type pascalien. On a admis comme une évidence fondamentale que les phénomènes physiques sont ordonnés selon des lois constantes. Puis on s'est efforcé d'intégrer les événements aléatoires, dont il fallait bien reconnaître l'existence, dans cette conception légaliste de l'expérience, en regroupant les événements aléatoires à l'intérieur de lois statistiques. Mais dans une telle conception les lois physiques restent de simples constatations empiriques. Nous ne pouvons pas leur trouver une explication par la seule réflexion, comme nous le pouvons pour les propriétés géométriques.

Or l'analyse critique de la philosophie humienne montre qu'avant la connaissance expérimentale il y a - tout au moins à l'état de virtualité - une pensée pré-expérimentale. C'est la pensée de Descartes réfléchissant sur la notion de Cogito. Nous faisons l'inventaire de toutes les pensées possibles et nous constatons que nous avons le pouvoir de nous représenter l'avenir sous une multitude infinie de formes différentes - en ce sens Hume applique une méthode spécifiquement cartésienne. Nous ne connaissons pas encore l'existence des lois expérimentales, mais nous sommes déjà capables, grâce aux liens que nous découvrons entre les idées, de démontrer la propriété de Pythagore.

Si l'on se reporte par l'imagination à ce moment de la pensée, logiquement premier sur le plan de la connaissance, l'ordre des problèmes se renverse. La succession aléatoire des résultats d'un jet de dé est expliquée directement par l'équivalence de ses six faces. Elle y trouve sa raison suffisante. Tout au contraire, c'est la répétition des mêmes événements qui exige une explication. Pourquoi quand nous lâchons dans le vide une bille d'acier, se trouve-t-elle régulièrement au même endroit après une seconde de chute, alors que nous pouvons aussi bien l'imaginer en mille endroits différents?

Dans une telle progression de la pensée le principe de raison suffisante n'apparaît plus comme un principe inconditionnel. Il a lui-même une genèse. Il est le produit de la contradiction entre l'ordre réel et le désordre imaginaire. Le réel devrait se présenter, selon l'expression kantienne comme « une rhapsodie de sensations ». Le fait qu'il n'en soit rien demande une explication, une raison suffisante de l'ordre perceptif. 


\section{L'existence du monde extérieur}

Les réflexions précédentes expliquent pourquoi il est impossible de fonder l'induction sur la seule expérience. Si grand que soit le nombre d'expériences au cours desquelles un événement s'est répété, il nous reste toujours possible d'imaginer mille événements différents, par exemple que notre bille d'acier se trouve après une seconde de chute dans une position différente de sa position habituelle.

Bien plus, en appliquant strictement le principe d'équiprobabilité à l'ordre perceptif, il est facile de prouver que celui-ci est en théorie infiniment improbable. Si nous supposons par exemple que nous pourrions apercevoir notre bille d'acier dans mille positions différentes, celles-ci doivent être considérées comme également probables et la probabilité qu'elle se retrouve exactement dans la même position doit être estimée à 1 sur 1000 .

En outre, nous retrouverons la même improbabilité à chaque expérience, de telle sorte que la probabilité de constater la présence de la bille dans une position identique au cours de $n$ expériences successives devrait être de 1 sur 1000 . En d'autres termes, elle serait pratiquement nulle.

Nous sommes ici dans la même situation qu'un joueur de dé jouant avec un tricheur. Supposons qu'à chaque jet de dé une somme d'argent soit mise en jeu, l'argent misé étant gagné par celui qui obtient le chiffre le plus fort et chacun des joueurs jouant avec son propre dé. Le tricheur obtient 6 cinquante fois de suite. Son adversaire n'hésitera pas à en conclure que le dé de son adversaire est pipé. Et, s'il a la possibilité de le vérifier, il constatera que son hypothèse est exacte. La probabilité d'un tel résultat est, en effet de 1 sur 6 et peut être considérée comme pratiquement nulle. Elle est cependant sans commune mesure avec la probabilité qu'un même événement se répète indéfiniment au cours d'expériences successives, s'il n'existe pas de raison cachée susceptible d'expliquer cette répétition.

La conclusion est claire : les données des sens ne sont pas de pures données de la conscience que l'on puisse considérer en elles-mêmes sans les expliquer par une réalité extérieure à elles. Assez curieusement le raisonnement de Hume établit et dément à la fois la conclusion à laquelle il aboutit. En montrant l'origine empirique de la notion de cause, Hume en interdit l'usage pour prouver l'existence d'une réalité extérieure. Mais en fondant sa démonstration sur l'équivalence du pensable et du possible, il aboutit par une toute autre voie à rendre cette même réalité intellectuellement nécessaire.

On comprend du même coup pourquoi une démonstration aussi simple a pu échapper pendant aussi longtemps à la perspicacité des philosophes. Elle va à contre-courant de la pensée classique. Pour celle-ci le seul raisonnement qui trouve sa justification en lui-même est le raisonnement déductif. Il s'impose directement à l'esprit grâce à sa propre évidence. Et la causalité est conçue pour expliquer la généralité des lois sur le modèle du raisonnement déductif : les causes sont supposées entraîner les effets par une nécessité 
interne du même type que celle qui lie les hypothèses et les conclusions. Ainsi la mécanique newtonienne explique-t-elle le mouvement des planètes à partir du principe d'inertie et de la gravitation. Ce mouvement peut être déduit more geometrico à partir de ces deux forces supposées.

En revanche le hasard est considéré comme essentiellement irrationnel parce qu'il paraît incompatible avec la nécessité des démonstrations géométriques. Ainsi s'efforce-t-on de retrouver à travers les événements aléatoires la généralité des lois physiques. Telle l'explication de Cournot qui voit dans les faits de hasard la rencontre de deux séries causales indépendantes. Le hasard est considéré comme ne pouvant fournir par lui-même un principe quelconque d'explication. L'opposition farouche au principe d'indéterminisme que nous retrouvons tout au long du $\mathrm{XX}^{e}$ siècle témoigne de la même conviction. Il paraît inacceptable de supposer dans le cours de la nature une part de hasard, si minime soit-elle.

\section{La nature du monde extérieur}

Le retour aux données immédiates de la conscience, au caractère aléatoire des perceptions, telles qu'elles nous sont directement connues, entraîne une conséquence capitale pour la philosophie de la connaissance. Il montre la nécessité d'admettre l'existence d'une réalité extérieure à la conscience ellemême et transcende la négation de la métaphysique.

En quoi consiste cette réalité, il serait présomptueux de répondre à une telle question, tout au moins dans l'état actuel de nos connaissances. Notre seule certitude est que cette réalité est « hors de la conscience», sans que nous puissions expliciter la nature des liens qui l'unissent à elle. Il est toutefois possible d'en donner une définition partielle en rappelant qu'elle a pour fonction d'expliquer les données sensibles. C'est ce que s'efforce de faire la science physique et d'une façon plus générale l'hypothèse de la matière. Comment imaginer une hypothèse plus efficace pour expliquer les résultats successifs d'une partie de dé que de supposer l'existence matérielle d'un cube homogène ? Là comme dans la démonstration géométrique explication et prévision sont indissociables. La seule explication valable des expériences passées est celle qui permet de prévoir le résultat des expériences futures. C'est le fait qu'elle ne permettait pas de prévoir le mouvement des astres qui a fait rejeter la théorie de l'Angelus rector. Mais on retrouve un caractère identique dans l'explication des régularités causales par les catégories de l'entendement. Elle ne permet pas des prévisions plus précises.

La thèse selon laquelle ce qui est également pensable doit être déclaré également possible conduit sans doute à modifier plusieurs conceptions de la connaissance qui sont largement répandues dans la pensée contemporaine. On 
peut donc supposer qu'elle rencontrera une assez vive résistance. Son seul argument est d'établir une plus grande cohérence entre les différents aspects de la pensée.

Séminaire d'Épistémologie,

Université d'Aix-Marseille III 